\title{
Estudo de um fluxo interno de materiais baseado na filosofia Lean Manufacturing
}

\author{
Alvaro Luiz Neuenfeldt Júnior ${ }^{a *}$, Julio Cezar Mairesse Silukª, Elpidio Oscar Benitez Nara ${ }^{\mathrm{b}}$ \\ a*Universidade Federal de Santa Maria, Santa Maria, RS, Brasil, alvjr2002@hotmail.com \\ bUniversidade de Santa Cruz do Sul, Santa Cruz do Sul, RS, Brasil
}

\begin{abstract}
Resumo
Nos últimos anos, é notória a evolução das empresas brasileiras no que tange a busca por otimizar os seus resultados industriais. Estudos do Lean Institute Brasil (2012) indicam que as indústrias do Brasil são, em média, as que necessitam o maior número de horas extras para o cumprimento das operações de manufatura, fato diretamente vinculado à elevada quantidade de estoques entre processos, desperdícios na movimentação e armazenagem de materiais. Logo, o artigo objetiva desenvolver uma metodologia que viabilize o estudo da maneira na qual funciona a cadeia de suprimentos interna de empresas fabricantes de ônibus do Brasil, em específico para os seus componentes comprados, com base no método denominado Making Materials Flow, para a correção das situações elencadas, havendo, ao final, uma redução em $40 \%$ do total de itens em falta para a linha de montagem e colaborando para o aumento de 37\% na produção por dia.
\end{abstract}

Palavras-chave

Lean Production. Manufatura de ônibus. Supply Chain Management.

\section{Introdução}

Atualmente as atividades logísticas são consideradas imprescindiveis para a determinação do nível de competitividade de uma empresa, mudando substancialmente as condicionantes dela quando de entregas atrasadas e na ausência de acurácia no controle dos materiais dos próximos processos. Em contrapartida, existem diversas formas empíricas de mudança que podem ser aplicadas para otimizar esse tipo de sistema, geralmente relacionadas a ações individuais promovidas pela força operária mas que, infelizmente, não disponibilizam aos envolvidos os recursos técnicos necessários para realizar-se a correta análise da situação encontrada e dos modos nos quais os itens estão sendo estocados, além da falta de consistência dos dados utilizados na busca da real causa-raiz dos problemas detectados (Jones \& Womack, 2003; Worley \& Doolen, 2006; Slack et al., 2008; Nasab et al., 2012; Neuenfeldt Júnior et al., 2014).

$\mathrm{Na}$ busca de vantagens competitivas perante o seu mercado de atuação, os processos de planejamento, implementação e controle do fluxo dos materiais da organização, em conjunto com o desenvolvimento integrado das informações a respeito dos componentes, são pontos fundamentais na sistematização dos meios de armazenagem e remessa, em tempo hábil, para a realização das ações de manufatura, a fim de tornar viável a redução do tack-time, ou tempo disponibilizado à manufatura de um ou mais itens da produção industrial (Ballou, 2003; Fullerton \& Wempe, 2009; Musetti \& Barbosa, 2011; Sanches et al., 2012).

Ballou (2003) e Emiliani (2010) destacam a necessidade do aumento de pesquisas científicas que demonstrem a relevância das tarefas sob responsabilidade da logística interna para as funções aplicadas da manufatura, focadas no produto final através da prestação de serviços no tempo certo, com as quantidades e qualidade desejadas, visando cada vez mais a otimização dos processos e das operações (Marx \& Paula, 2011; Chauhan \& Singh, 2012). Ainda, estudos do Lean Institute Brasil (2012) afirmam que o Brasil é um dos países que necessita do maior 
número de horas extras para o cumprimento de suas atividades industriais, fato vinculado principalmente à elevada quantidade de estoques e desperdícios na movimentação e armazenagem industrial.

De modo a mitigar tais problemas, a cadeia de suprimentos (supply chain) deve priorizar a implantação e manutenção de sistemáticas capazes de planejar e controlar esse fluxo, em conjunto com a integração com outros setores, contribuindo significativamente para a redução dos custos operacionais (Perez et al., 2006; Christopher, 2011; Musetti \& Barbosa, 2011; Bowersox et al., 2009).

Com esses conceitos, a filosofia Lean Manufacturing considera a análise sobre as rotinas de trabalho, a fim de identificar possíveis situações onde o processo não agrega valor para a produção, propondo a eliminação dessas atividades, a fim de restarem apenas as ações ou procedimentos pelos quais os clientes estão dispostos a pagar (Ohno, 1988, 2007; Jones \& Womack, 2004; Lopez et al., 2013).

Logo, é possivel afirmar que o objetivo da pesquisa é desenvolver uma metodologia que viabilize o estudo da maneira na qual funciona a cadeia de suprimentos interna para empresas fabricantes de ônibus do Brasil, em específico para os seus componentes comprados, de forma a aplicar ações capazes de remeter à obtenção de ganhos no sistema industrial como um todo, principalmente por esse ser caracterizado como de alta customização do produto final.

A proposta inicia-se com o levantamento das características estruturais atuais, indo ao encontro das informações consideradas relevantes para a próxima etapa, do desenvolvimento de um modelo matemático capaz de mostrar os parâmetros a serem seguidas no momento de se aplicar, na última fase da pesquisa, a nova sistemática de trabalho, acompanhada com a proposição de indicadores de mensuração do desempenho que possibilitem exprimir a situação antes e depois das alterações realizadas.

Inicialmente, assume-se que as pessoas envolvidas na cadeia de suprimentos não estão conscientes dos problemas existentes nos setores envolvidos, de modo a ser necessária a análise prévia do cenário atual, demonstrando quais são os principais pontos a serem trabalhados pela aplicação proposta.

\section{Metodologia}

Para abordar o problema é utilizado como referencial metodológico a pesquisa-ação, devido à relação entre o tema e as suas características práticas (Bradbury \& Reason, 2003; Groves et al., 2011). Além disso, por considerar a utilização básica de dados numéricos na sua resolução, é utilizado o método de pesquisa quantitativa, para possibilitar a identificação do quanto as variáveis adotadas impactam na problemática, evitando a produção de efeitos confusos e desconexos à abordagem utilizada.

Como base didática, foram analisadas as publicações de Rother \& Shook (1999), Shook \& Marchwinski (2003), Jones \& Womack (2003, 2004) e Harris et al. (2004) a respeito da filosofia Lean Manufacturing, predisposta para permitir o exame da situação em quatro partes: a primeira relata a análise do funcionamento do sistema anterior, através da caracterização do tema na situação encontrada e dos parâmetros que contribuem com maior relevância para o contexto, selecionando-os pela elaboração do método Análise das Discrepâncias proposto por Bandeira (2009), de modo a sintetizar os fatos que são mais relevantes.

Na segunda etapa houve o desenvolvimento dos indicadores para a mensuração da performance entre a situação anterior à obtida após as modificações, baseado em Slack et al. (2008), levando-se em consideração conceitos relacionados à otimização da produção e à falta de componentes no momento certo durante a montagem dos produtos finais. A seguinte fase foi idealizada a fim de compor a metodologia numérica para a correção das situações elencadas como prejudiciais, conforme os pressupostos descritos por Harris et al. (2004), através da pesquisa denominada por Making Materials Flow, e, finalmente, com as informações quantitativas a respeito do ideal é proposta a aplicação da nova estrutura e a verificação dos resultados.

Quanto à proposta prática, o estudo foi realizado na segunda maior fabricante de veículos coletivos do Brasil, de acordo com a Associação Nacional dos Fabricantes de Ônibus - FABUS (2012), com os materiais comprados pertencentes às suas duas linhas principais. Tal escolha deve-se à relação direta existente entre os pesquisadores e a empresa, bem como sua representatividade de mercado no que tange ao universo em que a organização está inserida. Os dados necessários foram compilados por meio de acesso a bancos de informação, documentos técnicos e diagnósticos semiestruturados aplicados a supervisores, gerentes e colaboradores da empresa.

\section{Análise do sistema anterior}

Inicialmente foram designados os setores vinculados com o supply chain dos componentes comprados, tais como: Almoxarifado, Logística Interna, Planejamento e Controle da Produção (PCP), Linha de Montagem e Estoque da Produção, sendo esses responsáveis por administrar mais de 3.900 itens, de 
300 fornecedores diferentes, distribuídos em 140 categorias distintas, e um giro de compras aproximado de 250 mil unidades por dia para, aproximadamente, 340 solicitações realizadas.

Historicamente, a cadeia de suprimentos foi baseada em um modelo que permitia a absorção de um alto volume de estoques, satisfazendo a demanda sem quaisquer faltas significativas no momento da montagem. Porém, nos últimos anos, houve duplicação da demanda, gerando assim efeitos colaterais como a falta de padronização dos locais de entrada e saída dos materiais estocados no Almoxarifado, contagens irregulares, danificação das peças no momento da estocagem e do transporte para a Linha de Montagem e queda no lucro da operação industrial que, em conjunto com diversas outras situações, resultaram em uma baixa organização das atividades de supply chain da empresa, gerando falta de comprometimento das partes envolvidas quanto ao cumprimento das metas estabelecidas.

Por esses motivos, nessa etapa é realizada a Análise das Discrepâncias existentes para os fatores considerados críticos de sucesso dos setores, através da comparação entre os desempenhos esperado e real dos parâmetros estabelecidos na matriz importânciadesempenho, concebida pela bibliografia baseada em Ballou (2003), Lambert (2008), Taylor (2008), Yiu et al. (2009) e Council of Supply Chain Management Professionals - CSCMP (2012). Sob essa óptica, com base em Bandeira (2009), a metodologia admite quatro aspectos na verificação:

a) Desenvolvimento de uma ideia concisa a respeito do que deve ser importante para que a função objetivo do sistema estudado ocorra eficazmente;

b) A avaliação de desempenho real do objetivo;

c) Foco no aprimoramento do desempenho por intermédio do pleno conhecimento das falhas existentes, dentre o que é importante, e o nível atual de desempenho; e d) Visualização e implementação dos planos de melhoria em decorrência da priorização de desempenho.

A elaboração da matriz importância-desempenho está estabelecida de acordo com a sugestão de dez critérios de verificação, identificados como: (A) Organização interna (condições em que se encontram disponibilizados os materiais no Almoxarifado); (B) Flexibilidade (capacidade estrutural de absorver as mudanças promovidas devido ao desenvolvimento de produtos novos); (C) Métodos gerenciais (formas de gestão da logística e estoques dos itens); (D) Planejamento e controle das operações (maneira com que os operadores estão dispostos e são requisitados, em analogia a descrição original das suas atividades); (E) Estrutura organizacional (verificação do modo como a matriz hierárquica de funções atende ao cumprimento das obrigações); (F) Fluxo dos componentes (existência de procedimentos operacionais padrões desde a chegada das peças até a sua aplicação na manufatura); (G) Fluxo da comunicação e informações (verificação de quais são os mecanismos utilizados para tal); (H) Liderança; (1) Risco e custos de recuperação (probabilidade de haver extravio de itens); e (J) Cultura e atitudes (fatores internos intrínsecos ao local em que a empresa está instalada).

Diante disso, os parâmetros foram estudados sob duas ópticas: quanto a sua importância para o desempenho organizacional e em relação ao que se considera ideal. Ambas as avaliações estão separadas em nove níveis, conforme a Tabela 1 .

A etapa final compreendeu do julgamento e avaliação de cada critério em comparação com os parâmetros estabelecidos, sendo possível localizá-los na matriz de importância-desempenho conforme a sua performance, a fim de se determinar os que necessitam ser verificados majoritariamente, segundo a Figura 1.

Em relação ao exposto, é visível que a priorização das medidas em curto prazo deve ser focada nos parâmetros destacados na zona Ação Urgente, onde há maior relevância dos itens identificados por Organização

Tabela 1. Parâmetros estabelecidos para a priorização dos critérios.

\begin{tabular}{|c|c|}
\hline Importância para o desempenho organizacional & Relação com ideal de performance dos critérios \\
\hline 1. Oferece vantagem crucial à competitividade & 1. Consideravelmente melhor que o idealizado \\
\hline 2. Proporciona vantagem importante & 11. Consistentemente melhor que o idealizado \\
\hline 3. Promove vantagem considerável & 111. Consistente e marginalmente melhor \\
\hline 4. Considera-se no nível de bom padrão & IV. Frequentemente melhor que as expectativas realizadas \\
\hline 5. Está na média do padrão estabelecido & V. Igual às expectativas \\
\hline 6. Fica a pouca distância da média & Vl. A pouca distância da média do sistema \\
\hline 7. Promissor, podendo ser um parâmetro importante no futuro & V11. Usual e pior que a média \\
\hline 8. Indiferente e muito raramente considerado & VIIl. Normalmente pior do que as expectativas \\
\hline 9. Irrelevante & 1X. Sempre pior \\
\hline
\end{tabular}

Fonte: Bandeira (2009). 


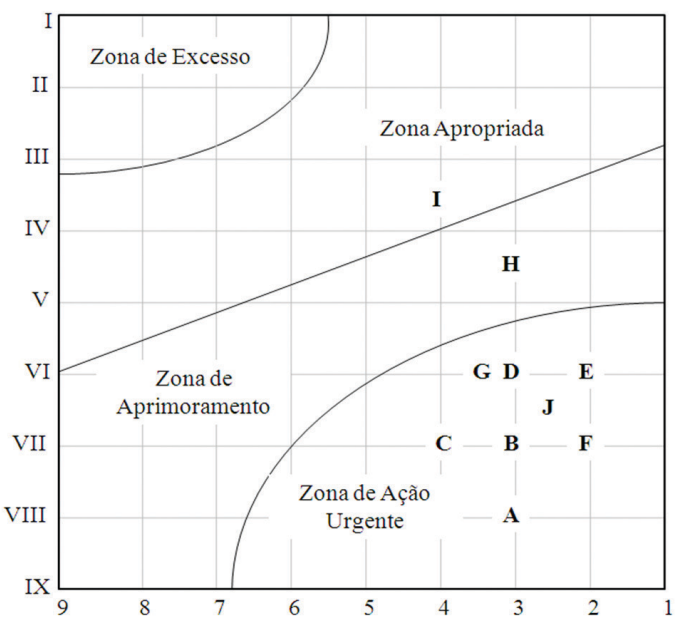

Figura 1. Priorização dos parâmetros conforme a sua situação.

interna (A), Flexibilidade (B) e Fluxo dos componentes (F), visando assim propor alterações que possibilitem impactar mais significativamente a empresa, focando na melhora dos resultados operacionais da manufatura. Em uma escala de importância inferior na zona Ação Urgente encontram-se (C), (G), (D), (J) e (E), de forma que esses estarão presentes nos estudos, porém de forma indireta e auxiliar à busca da otimização proposta.

Através desse balizamento, buscaram-se metodologias teórico-práticas capazes de direcionar os setores envolvidos para o aumento da manufatura de produtos acabados nas principais linhas de montagem. Diante disso, a seguir é apresentado o modelo de ação corretiva baseado na filosofia Lean Manufacturing.

\section{Mensuração da performance}

De acordo com a proposta de comparar o sistema estudado antes e depois das alterações sinalizadas, é necessário elaborar indicadores de performance capazes de expor as características e os fatores considerados relevantes para análise. Dessa forma, adotaram-se como referência base os pressupostos descritos por Slack et al. (2008) por meio da proposição de dois indicadores, onde o primeiro está constituído de maneira a revelar os efeitos das alterações diretamente nos produtos finais, denominado Média de ônibus finalizados, conforme descreve a Equação 1,

$$
A F B=\sum_{d=1}^{n} \frac{\left(U_{p}+R_{p}\right)_{d}}{n}
$$

sendo $\mathrm{U}_{\mathrm{p}}$ o número de ônibus urbanos produzidos, $\mathrm{R}_{\mathrm{p}} \mathrm{o}$ número de ônibus rodoviários produzidos e $d$ é o número de dias em cada mês, $\forall \mathrm{d} \in\{1,2, \ldots, \mathrm{n}\}$, com um alvo a ser atingido de $A F B(o b j)=12,00$, definido pela direção da empresa. 0 segundo indicador está vinculado com a eficiência na qual a remessa dos componentes é realizada para a Linha de Montagem, através da métrica denominada por Falta de componentes entregues, na qual pressupõe-se uma relação direta dele $\left(\mathrm{U}_{c_{w}}\right.$ para os urbanos e $\mathrm{R}_{c_{w}}$ para os rodoviários) com o total de requisições realizadas ( $\mathrm{U}_{\mathrm{c}_{t}}$ para os urbanos e $\mathrm{R}_{\mathrm{c}_{t}}$ para os rodoviários), por exemplo, ao dia, por intermédio da Equação 2,

$L P=\sum_{d=1}^{n} \frac{\left[\sum_{i=1}^{n}\left(\frac{U_{c w}}{U_{c t}}+\frac{R_{c w}}{R_{c t}}\right)\right]_{i}}{n}$

onde $i \in\{1,2, \ldots, n\}$ referencia os itens em estudo. Da mesma maneira que no caso anterior, o alvo foi definido pela direção em um total de $\mathrm{LP}(\mathrm{obj})=6,50$, conforme o que se julgou plausivel para um atendimento capaz de atender às exigências mínimas da manufatura.

\section{Ações corretivas}

Com a identificação dos problemas atuais, a modelagem definida propõe a verificação quantitativa das características do sistema, baseada em três etapas, a partir dos pressupostos descritos por Harris et al. (2004): (A) Desenvolvimento do plano para cada peça; (B) Construindo o mercado de peças; e (C) Elaboração das rotas de entrega, relatando transformações que vão desde o meio ao qual os produtos são estocados até as melhores práticas a serem adotadas na remessa deles.

\subsection{Desenvolvimento do plano para cada peça (DPCP)}

A utilização do DPCP visa a documentação das características básicas dos componentes, obedecendo a regra de que nenhum produto pode ser transferido dentro da empresa sem controle, diferentemente da situação encontrada anteriormente. Para o melhor dimensionamento das necessidades físicas da nova sistemática de armazenamento, o estudo parte, em um primeiro momento, da verificação de quanto espaço eles podem ocupar $(A R)$, possibilitando assim um posterior agrupamento dos valores obtidos nas categorias $(T A R)$, coerentemente com o nível de similaridade encontrado entre eles. Após isso, houve o agrupamento das categorias em concordância com o sistema de armazenamento mais conveniente ( $A S A)$, para assim se chegar ao valor final TSAR.

Contudo, os dados foram formatados através da coleta realizada nos setores de Planejamento e Controle da Produção, Almoxarifado, Linha de Montagem e Engenharia de Produto, estando organizados em 
planilhas eletrônicas, contemplando as informações básicas dos itens, como: código, descrição, unidade de dimensão, formato, local de armazenamento, local de utilização, uso diário, fornecedor, tamanho do lote padrão, pedidos por mês e prazo de entrega.

Partindo da necessidade do cálculo das características individuais de cada componente, Harris et al. (2004) relatam que esse tipo de informação é imprescindivel para tornar possível o entendimento do quanto de área eles necessitam, sendo uma das informações mais relevantes o seu nível de uso diário médio $(A C U)$ nos últimos seis meses, dado pela Equação 3,

$$
A C U=\sum_{d=1}^{n} \frac{C C_{d}}{n}
$$

onde CC é o consumo total diário encontrado, para então ser realizado o cálculo do tamanho do lote de fornecimento (LS), relativos aos seus padrões $A C U(L C)$, e mínimo característico $(M L P)$, conforme a Equação 4,

$$
L S=L C+M L P\left\{\begin{array}{l}
M L P \geq L C ; L C=0 \\
M L P<L C ; M L P=0
\end{array}\right.
$$

Devido a contratos comerciais em que o lote padrão é fixado, há situações em que a $L C$ não se aplica, pois $M L P \geq L C$, sendo necessário adotar apenas o $M L P$, o que resulta em estoques superiores a demanda, gerando assim discrepâncias que devem ser atenuadas por ações como a diminuição na frequência dos pedidos, alocação da diferença entre o $L C$ e $M L P$ no estoque do fornecedor ou renegociação até valor do $M L P$ se tornar igual ao valor de $L C$. Ao contrário, há situações em que a demanda do fornecedor é maior do que o previsto $(L C>M L P)$ e, portanto, se adota apenas a quantidade calculada para a dimensão do Almoxarifado. Consequentemente, a Equação 5 é definida de maneira que $P F$ é a frequência de entrega pelo fornecedor, $m$ é o número de meses do ano, utilizando-se no caso $m=6$, pois o horizonte semestral está de acordo com absorção do maior número de distorções nas vendas, e SS é o estoque mínimo que a empresa deve sempre manter em depósito independentemente da flutuação de vendas e do índice de consumo de cada item (UI).

$$
\begin{aligned}
& L C=\frac{\sum_{m=1}^{n} A C U_{m}}{n} * P F+S S ; \\
& S S=\left\{\begin{array}{l}
\frac{\sum_{m=1}^{n} A C U_{m}}{n} \geq U I ; \frac{\left(\sum_{m=1}^{n} A C U_{m}\right) *(1-\alpha) * 0.01}{n} \\
\frac{\sum_{m=1}^{n} A C U_{m}}{n}<U I ; \sqrt{\frac{\sum_{m=1}^{n} A C U_{m}}{n} * P F}
\end{array}\right.
\end{aligned}
$$

$$
\text { Quando } \frac{\sum_{m=1}^{n} A C U_{m}}{n} \geq U I \text {, o uso na produção é }
$$

considerado normal, e $\frac{\sum_{m=1}^{n} A C U_{m}}{n}<U I$ é definido quando o item pode ser considerado como de exceção ou uso não padrão. Para tanto, de acordo com Ballou (2003), $\alpha \in\{0 \%, \ldots, 100 \%\}$ é o fator de segurança, que varia de acordo com o seu nível de criticidade, relacionando desde confiabilidade do fornecedor, precisão de inventário, frequência de uso, importância em caso de falhas, dentre outros. Com isso, é possivel calcular o quanto de área $\left(A R_{i}\right)$ os componentes precisam para serem armazenados corretamente, de acordo com a Equação 6, além de definir-se o valor necessário para as diversas categorias elencadas (TAR), compatível com a Equação 7,

$$
\begin{aligned}
& A R_{i}=W_{i} * L_{i} *\left(\frac{L S}{Q}\right) \\
& T A R_{y}=\sum_{i=1}^{n} A R_{i}
\end{aligned}
$$

onde $y$ representa a numeração das categorias e $i$ está relacionado ao número de itens classificados em cada uma delas, $W_{i}$ e $L_{i}$ são as variáveis que representam, respectivamente, o comprimento e a largura do pacote, de acordo com a variável $Q$, que define o número total de peças que o pacote pode conter. Analogamente ao supracitado, foram analisados 3.900 elementos ao longo da cadeia de suprimentos $(i=3900)$, de maneira que, de acordo com o seu grau de similaridade, foram separados em 140 categorias $(y=140)$. Porém, o dimensionamento do Almoxarifado foi condicionado aos tipos $(x)$ de sistema de armazenamento levantados anteriormente a essa pesquisa, somando um total de 22 possibilidades $(x=22)$, sendo necessário para o cálculo da sua área $\left(\mathrm{SAR}_{\mathrm{x}}\right)$ dimensionar o espaço disponibilizado nos $x$ modos de estocagem (ASA), conforme prevê a Equação 8,

$$
A S A_{x}=\frac{H_{x}}{H s_{x}} *\left(W_{x} * L_{x}\right)
$$

para $\mathrm{H}_{\mathrm{x}}$ definido como a altura utilizada, $\mathrm{W}_{\mathrm{x}}$ e $\mathrm{L}_{\mathrm{i}}$ definidos, respectivamente, como o seu comprimento e largura e $\mathrm{Hs}_{x}$, como a medida dos vãos existente. Com isso é possível verificar o valor de $\mathrm{SAR}_{x}$, pela Equação 9:

$$
S A R_{x}=\frac{1}{A S A_{x}} * \sum_{y=1}^{n}\left(W_{x} * L_{x}\right) * T A R_{y}
$$

Para a área de estocagem necessária no Almoxarifado, se faz imprescindível o cálculo do quanto de espaço, em proporção a SAR, o corredor, o recebimento e a expedição dos materiais necessitam 
ocupar. Tompkins \& Smith (1998) e Neapolitan (2003) definem, para as características de armazenamento em estudo, que essa é igual a $100 \%\left(\delta_{t}=1\right)$, onde $10 \%$ do espaço deve ser ocupado pela expedição $\left(\delta_{\mathrm{e}}=0,10\right)$, $15 \%$ por atividades de recebimento $\left(\delta_{r}=0,15\right), 40 \%$ pelos corredores $\left(\delta_{c}=0,40\right)$ e, finalmente, $35 \%$ pelos estoques em si $\left(\delta_{\mathrm{pa}}=0,35\right)$. Com isso, é possível o cálculo final de TSAR, dado na Equação 12,

$$
\begin{aligned}
& T S A R=\delta_{t} * \sum_{x=1}^{n} S A R_{x} \\
& T S A R=\left(\frac{\delta_{p a}}{\delta_{p a}}+\frac{\delta_{c}}{\delta_{p a}}+\frac{\delta_{r}}{\delta_{p a}}+\frac{\delta_{e}}{\delta_{p a}}\right) * \sum_{x=1}^{n} S A R_{x} \\
& T S A R=\left(\frac{0,35}{0,35}+\frac{0,40}{0,35}+\frac{0,15}{0,35}+\frac{0,10}{0,35}\right) * \sum_{x=1}^{n} S A R_{x}
\end{aligned}
$$

proposto através da colocação em evidência da variável $\mathrm{SAR}_{x}$ em conjunto com os valores das proporções de cada um dos elementos básicos considerados.

Como o cálculo da área necessária total está diretamente relacionado à construção de cenários baseados na demanda produtiva, principalmente para a estimativa de vendas dos próximos anos, realizaram-se três análises. A primeira relata a expectativa utilizando o potencial total da fábrica (cenário otimista: 12 ônibus por dia), o segundo baseia-se na utilização relativamente média da capacidade (cenário normal: 10 ônibus por dia) e o terceiro está formatado para uma baixa de produção (cenário pessimista: 9 ônibus por dia). Com esses dados, a conclusão a que se chega é que o total necessário para o cenário otimista é $T S A R=2.732 \mathrm{~m}^{2}$, em uma área de estocagem de $S A R=956 \mathrm{~m}^{2}$.

Comparado com o antigo sistema, que ocupava $3.500 \mathrm{~m}^{2}$, houve a otimização de $22,8 \%$ do espaço, possibilitando assim o seu aproveitamento para outros fins industriais. Além disso, foi possivel obter um melhor dimensionamento da ocupação proporcional do ambiente por parte dos elementos básicos presentes em um Almoxarifado, alocando-se melhor principalmente o recebimento de materiais, com ganho de aproximadamente $20 \%$ de espaço, e corredores (15\% a mais), o que possibilitou ganhos no arranjo das atividades de controle, armazenamento e picking dos componentes.

\subsection{Construindo o mercado de peças}

Logo após a realização dos cálculos de redimensionamento é necessário verificar a forma como materiais serão estocados, de maneira a adotar novas técnicas capazes de atender mais eficazmente a organização do contexto.
Inicialmente, verificou-se que a empresa não necessita de dois espaços distintos, sendo realizado, por consequência, o fechamento do Almoxarifado 2. Esse foi estabelecido por meio de estudos de viabilidade técnica e econômica em que, para o primeiro utilizou-se das percepções dos operadores e supervisores acerca do formato de trabalho aplicado, no qual se destacou a dificuldade de se atender a tempo as requisições de remessa devido à distância existente entre eles. A seguir foram propostos cálculos capazes de determinar a taxa de armazenamento do local, segundo as proposições de Yamashiro (2005), a fim de se encontrar a diferença do anterior em analogia à proposta. Por questões mercadológicas, não foi possível identificar os valores obtidos através do cálculo realizado, no entanto chegou-se à conclusão de que a antiga sistemática era 15\% mais cara, por ano, do que a operação centralizada. Diante dos fatos estabelecidos, optou-se por centralizar os almoxarifados, escolha viabilizada pela expansão do Almoxarifado 1.

0 segundo passo determinou o novo regime de controle dos estoques. Para isso definiu-se como nova sistemática de manuseio dos materiais a chamada FIFO (primeiro a chegar, primeiro a sair), usando um corredor central amplo capaz de atender à alta demanda de movimentação tanto de entrada quanto de saída, evitando-se assim a possibilidade de interrupções na estocagem ou na movimentação para a Linha de Montagem.

\subsection{Elaboração das rotas de entrega}

Com os componentes organizados em um armazém central e unificado é necessário determinar a forma de movimentação deles, alternando um sistema de abastecimento baseado em carregamento de itens individualizados para um formato de kits, através de contentores, transportados por tratores, contendo todos os itens pertencentes às estações de trabalho, em relação ao produto que está programado para ser manufaturado.

Dessa forma, a composição dos kits é realizada no Almoxarifado, com um espaço específico representativo das estações de trabalho, e eles são fornecidos em intervalos de tempo predeterminados, havendo, para as Linhas de Montagem, rotas de entrega específicas, sincronizadas conforme o período de fabricação dos ônibus, a fim de evitar possíveis faltas de componentes. 0 processo foi organizado por meio do cumprimento de quatro etapas: preparação $(P R)$, remessa $(D)$, estoque preliminar (ST) e uso (US), determinadas pelas Equações 13, 14, 15 e 16, com base nos pressupostos de Jones \& Womack (2004): 
$P R(k)=T+(k+2)$

$D(k)=T+(k+1)$

$S T(k)=T+k$

$U S(k)=T+(k+1)$

de maneira que $T$ é o tempo decorrido durante um dia de expediente e $k$ é a variável que identifica qual kit está sendo calculado. Cada estação de trabalho possui três locais para colocação dos contêineres: dois de estoque (ST) e um para uso (US), quando, supostamente, o kit 1 for usado $(T+2)$, o setor de Logística Interna estará posicionando no primeiro local de estoque o kit 3, que será utilizado apenas em $T+4.0$ kit 2 (entregue em $T+1$ ) já estará em espera pronto para uso e, por sequência lógica, o kit 4 está no processo de elaboração no Almoxarifado (usado em $T+5)$. Com isso, é possível mitigar com mais agilidade potenciais faltas, através da disponibilização de um tempo maior para a constatação do problema.

Com a realização da sincronização é necessário determinar a frequência com que ocorre a entrega dos componentes, partindo-se do estabelecimento da expectativa média de demanda dos dois tipos de produtos $\left(\mathrm{D}_{\mathrm{avr}}\right)$, dada na Equação 17,

$$
D_{a v r}=\sum_{m=1}^{n} \frac{U_{m}}{n}+\sum_{m=1}^{n} \frac{R_{m}}{n}=U_{a v r}+R_{a v r}
$$

onde $\mathrm{U}_{m}$ representa os ônibus urbanos e $\mathrm{R}_{\mathrm{m}}$, os rodoviários, com $\mathrm{U}_{\text {avr }}$ e $R_{\text {avr }}$ sendo os valores médios de ambos, originados a partir do somatório da média de carros produzidos por período considerado. Como a remodelação do Almoxarifado foi realizada para uma expectativa de 12 ônibus/dia, a sua estruturação se predispôs de acordo com a análise previamente realizada em relação a essa expectativa, levando em consideração as vendas dos últimos dois anos $(m=24)$, na qual demonstraram-se valores médios de $\mathrm{U}_{\text {avr }}=8$ e $R_{\text {avr }}=4$. Com isso é possível calcular a razão de proporção existente entre essas duas variáveis $\left(\mathrm{P}_{\text {avr }}\right)$, conforme a Equação 18,

$$
P_{a v r}=\frac{R_{a v r}}{U_{a v r}}
$$

para tack-time dos rodoviários duas vezes superior ao dos urbanos $\left(\mathrm{P}_{\mathrm{avr}}=2\right)$, consequência das diferentes complexidades de montagem existentes - a primeiro visa uma maior lucratividade por unidade e a segundo, maiores volumes por lote remetido ao cliente. Com isso, os kits dos urbanos são deslocados a cada 30 minutos e os do rodoviário de hora em hora.

\section{Nova estrutura de trabalho e resultados}

A adoção da nova metodologia foi realizada durante um período de dois meses $(P 2)$, estabelecida sob o regime de organização em que há uma forte dependência conjunta na forma com que os setores colaboraram para a efetiva realização das alterações.

Diante desse fato, primeiramente a linha de Montagem foi separada por estações de trabalho, com tarefas padronizadas e avanço de produção normalizado. Alguns setores, como o Almoxarifado, a Logística Interna e Estoque da Produção sofreram grandes mudanças em suas atividades e estrutura para adequarem-se às novas diretrizes estabelecidas na seção anterior, sendo o último setor o mais afetado: foi extinto. Por fim, o PCP não exigiu mudanças substanciais em sua dinâmica, devido a atual estrutura já ser considerada ideal, havendo pequenas alterações na interrelação das pessoas dele com as dos citados anteriormente.

Após os dois meses iniciais, experimentou-se um processo chamado de mudança cultural $(P 3)$, onde 0 foco estava nas pessoas envolvidas no supply chain, a favor da adequação dos operadores transferidos a outros departamentos, e na conscientização sobre os novos métodos aplicados. Superada essa fase, tem-se o que se considera o sistema plenamente desenvolvido $(P 4)$, gerado a partir da expectativa de pleno funcionamento do modelo. Assim, a fim de medir a evolução das mudanças durante todos esses períodos, em comparação ao estado inicial $(P 1)$, apresenta-se a utilização dos indicadores de eficiência propostos, iniciando-se pela média de ônibus finalizados por dia $(A F B)$ em concordância ao mostrado na Figura 2.

Nota-se que em $P 1$ houve a produção média de 7,60 ônibus por dia, já em $P 2$ obteve um significativo crescimento de $36 \%$, passando para $\overline{\mathrm{AFB}}(\mathrm{P} 2)=10,00$, o que não se confirmou durante o período de mudanças culturais, com queda de 3\% do indicador $(\overline{\mathrm{AFB}}(\mathrm{P} 3)=9,69)$, a qual, apesar de ser pequena, contrastou com o aumento de volume esperado. Finalmente, na implantação $(P 4)$ encontrou-se a média de $\overline{\mathrm{AFB}}(\mathrm{P} 4)=10,67$ ônibus manufaturados por dia, um aumento em relação a $P 1$ de $40,40 \%$, comparado ao de $10,11 \%$ da $P 2$, o que impossibilitou o cumprimento do objetivo estabelecido $(\mathrm{AFB}(\mathrm{obj})=12,00)$. No segundo indicador, definido para mensurar o índice de falta de componentes entregues $(L P)$ à Linha de Montagem, houve uma significativa redução dos valores desde $P 1$ até $P 4$, com queda de 38,97\% de faltas das peças, de acordo com a Figura 3.

É perceptível que as alterações realizadas resultaram em mudanças substanciais dos problemas de abastecimento de peças, fato esse confirmado 


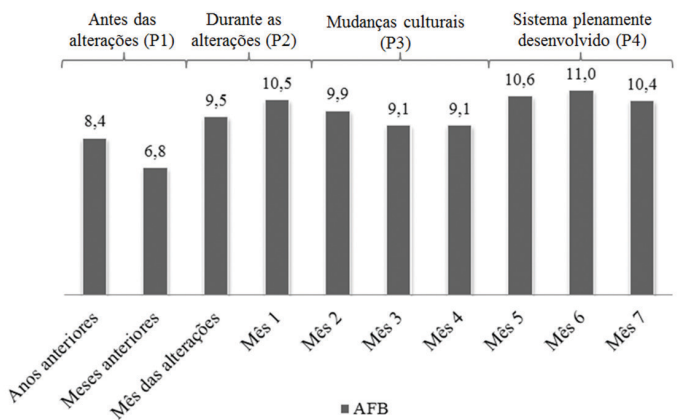

Figura 2. Produção media calculada ( $A F B)$.

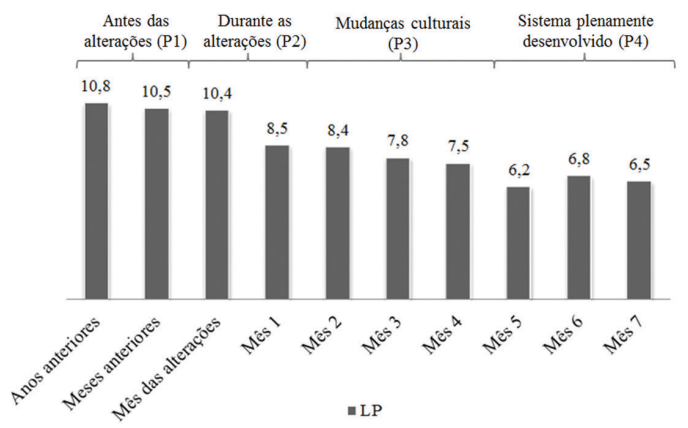

Figura 3. Falta de peças calculada $(L P)$.

quando comparados os resultados anteriores $(10,8)$ aos atingidos em $P 4, \mathrm{LP}(\mathrm{P} 4)=6,50$, com o objetivo estabelecido de $\mathrm{LP}(\mathrm{obj})=6,50$, apesar da sinalização de um novo aumento das faltas detectado pelos valores obtidos nos meses 6 e 7 , se comparados com o mês 5 .

De maneira geral, pode ser indicado que as melhorias não foram obtidas apenas com a aplicação dessa metodologia, mas sim através do conjunto de diversas outras atividades internas ocorridas em cada um dos setores envolvidos, tais como: estratificação das estações de trabalho em subníveis, facilitando o desenvolvimento de procedimentos operacionais padrões a partir do modelo de carro em processo operativo; atualização e construção de novas estruturas de produto digitais, a partir de uma força tarefa entre o PCP e a Engenharia de Produto; ações de $5 \mathrm{~S}$ e remodelagem do layout de manufatura para os departamentos denominados Fabricação e Plásticos; e a remodelação do padrão de avaliação dos fornecedores internos e externos.

Em comum, tais ações visaram o aumento da eficiência da produção e do número de vendas durante e após os períodos estabelecidos para as modificações, observando-se também uma redução de aproximadamente 30\% no número de horas extras necessárias que, em média, beiravam as 800 horas semanais. Como consequência, houve a realocação de trabalhadores da montagem para outros locais da indústria, resultado da eliminação do turno noturno para a linha de ônibus rodoviários.

\section{Conclusões}

Com as experiências do estudo aconteceram aprendizados relevantes a respeito da importância de se mudar uma forma de trabalhar que estava em desacordo com as expectativas de mercado. Apesar dos bons resultados obtidos, ocorreram diversas dificuldades na implantação do novo sistema nos primeiros meses, principalmente para manter a organização do Almoxarifado central na troca dos processos de controle das peças, o que, apesar de ser um empecilho, era esperado pelas partes envolvidas com a implantação.

Em conjunto, a análise dos dados quantitativos e qualitativos durante os períodos de aplicação da metodologia foi fundamental para se monitorar os ganhos na organização do material, espaço físico e qualidade de trabalho das pessoas, resultando, por consequência, na redução dos tempos de fabricação do produto final. No entanto não foi possível, após sete meses da implementação, atingir a meta definida para 0 $A F B$, principalmente devido a fatores que não estavam diretamente relacionados às atividades de supply chain mas a gargalos de produção relacionados a questões do processo produtivo na Linha de Montagem.

A respeito do objetivo estabelecido, de desenvolver uma metodologia que viabilizasse o estudo da maneira na qual funciona a cadeia de suprimentos interna de empresas fabricantes de ônibus do Brasil, em específico a dos seus componentes comprados, pode-se afirmar que a aplicação do modelo adotado atendeu à proposta, de modo a remeter, após a aplicação de ações de melhoria, à otimização das ações envolvidas, contribuindo diretamente para os gradativos aumentos da produção mensurados.

Algumas limitações foram encontradas no decorrer da pesquisa, como a dificuldade na obtenção dos dados necessários sobre os itens, devido principalmente à alta customização do produto final, o que acarretou uma grande quantidade de obsoletos ou inexistentes, mas que não podem ser eliminados por questões de pós-vendas. Além disso, houve dificuldades na sincronização das informações sobre a nova proposta entre os setores industriais, o que dificultou as relações de trabalho principalmente nas etapas $P 2$ e $P 3$.

Para futuras pesquisas, os autores sugerem a aplicação do modelo estudado ao restante da cadeia de fornecimento (componentes fabricados, remanufaturados e mistos), promovendo-se assim a padronização da forma de controle, armazenagem e abastecimento de materiais. Outra sugestão é aplicar 
o método a outros contextos de fabricação, como aqueles usados pelos mais relevantes fornecedores externos, permitindo ganhos produtivos não somente em um dos lados do sistema, mas de maneira integrada, para todas as partes envolvidas.

\section{Referências}

Associação Nacional dos Fabricantes de Ônibus - FABUS. (2012). Produção. Retrieved from http://www.fabus.com. br/pro-ducao.htm

Ballou, R. H. (2003). Business logistics: supply chain management. Upper Saddle River: Prentice Hall.

Bandeira, A. A. B. (2009). Performance indicators. Rio de Janeiro: Qualitymark.

Bowersox, D. J., Closs, D. J., \& Cooper, M. B. (2009). Supply chain logistics management. New Jersey: McGraw-Hill.

Bradbury, H., \& Reason, P. (2003). Action research: an opportunity for revitalizing research purpose and practices. Qualitative Social Work, 2(2), 155-175. http:// dx.doi.org/10.1177/1473325003002002003

Chauhan, G., \& Singh, T. P. (2012). Measuring parameters of lean manufacturing realization, Measuring Business Excellence, 16(3), 57-71. http://dx.doi. org/10.1108/13683041211257411

Christopher, M. (2011). Logistics and supply chain management. New Jersey: FT Press.

Council of Supply Chain Management Professionals - CSCMP. (2012). Supply chain management: logistics definitions. Retrieved from http://www.cscmp.org/website/ aboutCSCMP/Definitions/Definitions.asp

Emiliani, B. (2010). Real lean: strategies for lean management success. Wethersfield: Center for Lean Business Management.

Fullerton, R. R., \& Wempe, W. F. (2009). Lean manufacturing, non-financial performance measures, and financial performance. International Journal of Operations \& Production Management, 29(3), 214-240. http://dx.doi. org/10.1108/01443570910938970

Groves, R. M., Fowler, F. J., \& Couper, M. P. (2011). Survey methodology. San Francisco: Wiley.

Harris, R., Harris, C., \& Wilson, E. (2004). Making materials flow. Cambridge: Lean Enterprise Institute.

Jones, D., \& Womack, J. (2003). Lean thinking: banish waste and create wealth in your corporation. New York: Free Press.

Jones, D., \& Womack, J. (2004). Seeing the whole value stream. Cambridge: Lean Enterprise Institute.

Lambert, D. M. (2008). Supply chain management: processes, partnerships, performance. Sarasota: Supply Chain Management Institute.

Lean Institute Brasil. (2012). Lean thinking. Retrieved from http://www.lean.org.br/o_que_e.aspx

Lopez, P. R. A., Santos, J. F., \& Arbós, L. C. (2013). Lean manufacturing: costing the value stream. Industrial
Management \& Data Systems, 113(5), 647-668. http:// dx.doi.org/10.1108/02635571311324124

Marx, A. M., \& Paula, 1. C. (2011). Proposta de uma sistemática de gestão de requisitos para o processo de desenvolvimento de produtos sustentáveis. Revista Produção, 21(3), 417-431. http://dx.doi.org/10.1590/ S0103-65132011005000041

Musetti, M. A., \& Barbosa D. H. (2011). The use of performance measurement system in logistics change process: proposal of a guide. The International Journal of Productivity and Performance Management, 60, 339359. http://dx.doi.org/10.1108/17410401111123526

Nasab, H. H., Bioki, T. A., \& Zare, H. K. (2012). Finding a probabilistic approach to analyze lean manufacturing. Journal of Cleaner Production, 29-30(9), 73-81. http:// dx.doi.org/10.1016/j.jclepro.2012.02.017

Neapolitan, M. (2003). The time, space and cost guide to better warehouse design. Boonton: Distribution Group.

Neuenfeldt Júnior, A. L., Siluk, J. C. M., Soliman, M., \& Marques, K. F. S. (2014). Study to evaluate the performance development of Brazilian franchise segments. Independent Journal of Management \& Production, 5(2), 381-397.

Ohno, T. (1988). Toyota production system: beyond large scale production. Cambridge: Productivity Press.

Ohno, T. (2007). Workplace management. New York: Gemba Press.

Perez, L. V., Bossio, G. R., Moitre, D., \& Garcia, G. O. (2006). Supervisory control of a hev using an inventory control approach. Latin American Applied Research, 36, 93-100.

Rother, M., \& Shook, J. (1999). Learning to see. Cambridge: Lean Enterprise Institute.

Sanches, J. H., Contador, J. C., \& Contador, J. L. (2012). A study of the competitiveness of the most important compact automotive vehicles manufactured in Brazil. Revista Produção, 22(1), 99-114.

Shook, J., \& Marchwinski, C. (2003). Lean lexicon. São Paulo: Lean Institute Brazil.

Slack, N., Chambers, R., Johnston, R., \& Betts, A. (2008). Operation and process management: principles and practice for strategic impact. Lebanon: Prentice Hall.

Taylor, G. D. (2008). Introduction to logistics engineering. Boca Raton: CRC Press. http://dx.doi. org/10.1201/9781420088571

Tompkins, J. A., \& Smith, J. D. (1998). The warehouse management handbook. Raleigh: Tompkins Press.

Worley, J.M., \& Doolen, T.L. (2006). The role of communication and management support in a lean manufacturing implementation. Management Decision, 44(2), 228-245. http://dx.doi.org/10.1108/00251740610650210

Yamashiro, A. (2005). The stock administration book. Oakland: National Center of Employee Ownership.

Yiu, K. F. C., Xie, L. L., \& Mak, K. L. (2009). Analysis of bullwhip effect in supply chains with heterogeneous decision models. Journal of Industrial and Management Optimization, 5(1), 81-94. http://dx.doi.org/10.3934/ jimo.2009.5.81 


\title{
Study of an internal materials workflow based on the Lean Manufacturing philosophy
}

\begin{abstract}
Recently, Brazilian companies have searched for ways to optimize the results of industrial processes. Studies by the Lean Institute Brasil (2012) indicate that the industries of Brazil, on average, require the most overtime for compliance with manufacturing operations. This is directly linked to the high amount of stocks between processes, waste handling and the storage of materials. Thus, this article aims to study the way in which the internal supply chain operates in a bus manufacturing company in Brazil. Specifically, purchased components are studied to find ways to obtain industrial gains, and a methodology called Making Materials Flow is used to correction the problems that are identified. In the end, missing items in the Assembly line were reduced by $40 \%$, the number of buses produced per day increased by approximately $37 \%$.
\end{abstract}

Keywords

Lean Production. Bus manufacturing. Supply Chain Management. 